\title{
Rapidly growing abdominopelvic mass - to be or not to be, not all we see is what it seems
}

\author{
Massa abdominopélvica de crescimento rápido - ser ou não ser, nem tudo o que vemos \\ é o que parece
}

Renata Sofia Fernandes Teixeira Veríssimo*1, Joana Mafalda Aidos ${ }^{1}$, Ana Isabel Sousa², Nuno Francisco Nogueira Martins²

\begin{abstract}
The finding of a pelvic mass is often a source of great concern to our patients. Establishing the correct diagnosis and accurately staging these tumors is mandatory, but sometimes difficult, as abdominal masses may prove to be benign or malignant and have different etiologies. We describe a patient with a rapidly growing abdominal mass with weight loss and other accompanying symptoms, that together with the exams performed, posed a genuine diagnostic challenge to the team. Only after surgery the authors reached the definitive diagnosis of leiomyoma. Still waters run deep.
\end{abstract}

Keywords: Leiomyoma. Sarcoma. Pelvic Mass.

\section{RESUMO}

O achado de uma massa pélvica constitui frequentemente uma preocupação para as nossas pacientes. Estabelecer o correto diagnóstico e o estadiamento destes tumores com precisão é mandatório, mas por vezes difícil, uma vez que as massas abdominais podem ser benignas ou malignas e ter diferentes etiologias. Apresentamos um caso de crescimento rápido de um tumor abdominal associado a perda de peso e outros sintomas, que em conjunto com os exames realizados colocaram um desafio à equipe. Somente após a cirurgia os autores confirmaram o diagnóstico definitivo de leiomioma. As aparências iludem.

Palavras-chave: Leiomioma. Sarcoma. Massa Pélvica.

1. Trainnee in Obstetrics and Gynecology at Centro Hospitalar Tondela-Viseu, Portugal

2. Consultant of Obstetrics and Gynecology at Centro Hospitalar Tondela-Viseu, Portugal
Corresponding: Renata Sofia Fernandes Teixeira Veríssimo Urbanização Tamonte, Rua João de Barros no 67 3030-388 - Coimbra - Portugal

Recebido em 03/04/2017 Aprovado em 31/08/2017 


\section{Introduction}

There are a number of disorders that may cause an abdominal-pelvic mass. Some are common, whilst others are quite unusual or even rare. The most prevalent uterine tumours are leiomyomas. Most of them are detected in middleaged women ( $e^{\prime \prime} 30$ years old). ${ }^{1}$

The incidence of endometrial cancer and uterine sarcomas is about 25 per 100,000 and 0.7 per 100,000 women, respectively. ${ }^{2}$

On the other hand, pelvic masses may become so enlarged that they might become palpable on either the pelvic or abdominal exams. Although such cases are often benign and tumors can become quite large, in other cases they can be malignant.

The finding of a pelvic mass is often a source of great concern to our patients. It is of the utmost importance to conduct a proper diagnostic investigation, relying on patient history, physical examination, laboratory tests and imaging techniques. In some cases, patients may even require a surgical diagnostic evaluation. Establishing a pre-operative accurate diagnosis and, if applicable, thoroughly staging these tumors is important, particularly when surgical resection might be an option.

\section{Case presentation}

A 46-year-old woman presented to the emergency department with anuria and abdominal discomfort. There was also history of bilateral oedema of the lower limbs, an increase of the abdominal volume over the previous few months and weight loss (about $4 \mathrm{~kg}$ in two months). The patient didn't have any gastro-intestinal complaints, including nausea, vomiting or diarrhoea.

Menarche was at 12 years old and she denied any abnormal vaginal bleeding. She was nulliparous and used barrier methods for contraception. Her past medical and surgical history were non-contributory, except for being a smoker of two cigarettes per day.

At the physical examination, the patient presented with a painless but markedly distended abdomen, slightly tender on palpation. A stony fixed abdominal mass of about $40 \mathrm{~cm}$ was palpable. She also had an umbilical hernia and slight abdominal collateral vascular circulation.
An abdominal scan revealed hepatomegaly with a heterogeneous central part; a voluminous and vascularized abdominal-pelvic mass appearing to have uterine origin and a moderate amount of intrabdominal free fluid. Blood workup was negative for hepatitis, but revealed a discrete increase of the gamma-glutamyl transferase, and the ROMA (Risk of Ovarian Malignancy Algorithm) was 9.7\% (high risk). Thorax x-ray showed a rise in both diaphragmatic domes and the abdominal $x$-ray was normal. A second sonographer performed a scan to control a paracentesis, and then found features compatible with liver metastization from a large abdominal solid mass arising from the right adnexa, with about $12 \mathrm{~cm}$, and the possibility of an ovarian cancer was suggested. The study of the ascitis was negative for neoplastic cells. The patient additionally performed an echo-doppler ultrasound to exclude Budd Chiari syndrome and a computerized tomography abdominal scan which revealed an abdominal-pelvic mass measuring $37 \mathrm{~cm} \times 26 \mathrm{~cm} \times 21 \mathrm{~cm}$, bilobulated, well delimitated in the abdominal part and irregular in the pelvis, with some coarse calcifications and necrotic areas suspicious of uterine sarcoma (figure 1 and 2). A guided-biopsy of the mass was then decided and performed and the histological results excluded malignancy and suggested the presence of leiomyoma.

The case was discussed at the Tumor Board and a decision to perform an exploratory laparotomy was reached.

At the midline laparotomy, a $42 \times 25 \times 30 \mathrm{~cm}$ uterus was found (figure 3 ), including a posterior fibroid of about $10 \mathrm{~cm}$ hosted in the Douglas pouch, and a giant fibroid arising from the fundus of the uterus with $20 \times 20 \mathrm{~cm}$, with light adhesions to the omentum. Right adnexa presented a $6 \mathrm{~cm}$ simple cyst and there was about $500 \mathrm{cc}$ of free ascitis. The liver presented a blue lesion on the surface of the right lobe. There weren't any other pathological findings on inspection or palpation, either on the abdomen or the pelvis. An hysterectomy with bilateral adnexectomy, collection of ascitic fluid and repair of the umbilical hernia were performed.

Gross pathologic examination revealed a specimen weighing $7040 \mathrm{~g}$ and measuring $10 \mathrm{~cm}$ of uterine corpus; arising from the uterus, there were 2 large nodules, the bigger with $22 \mathrm{~cm}$ and other several smaller nodules some of them with 


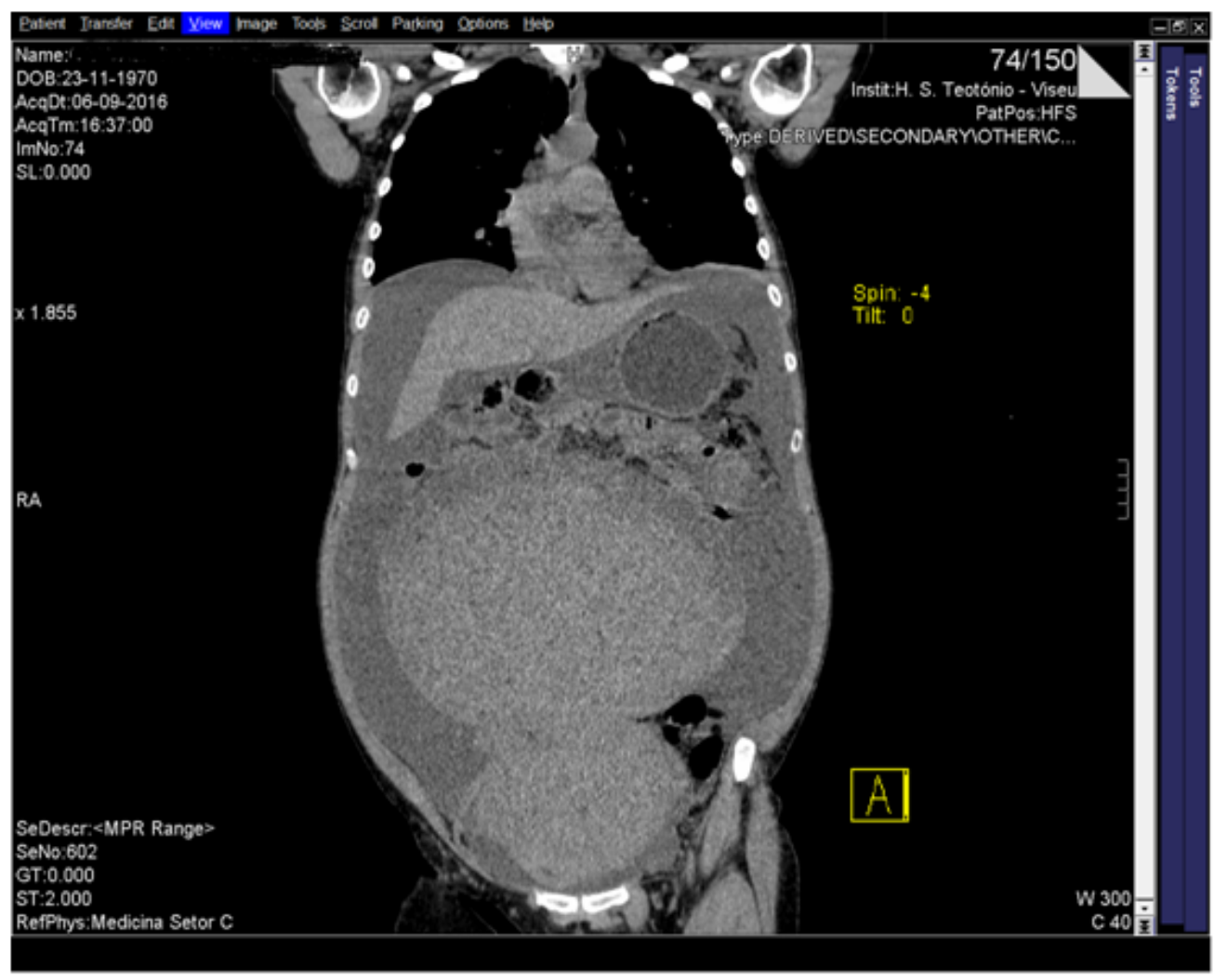

Figure 1: CT scan (sagital view)

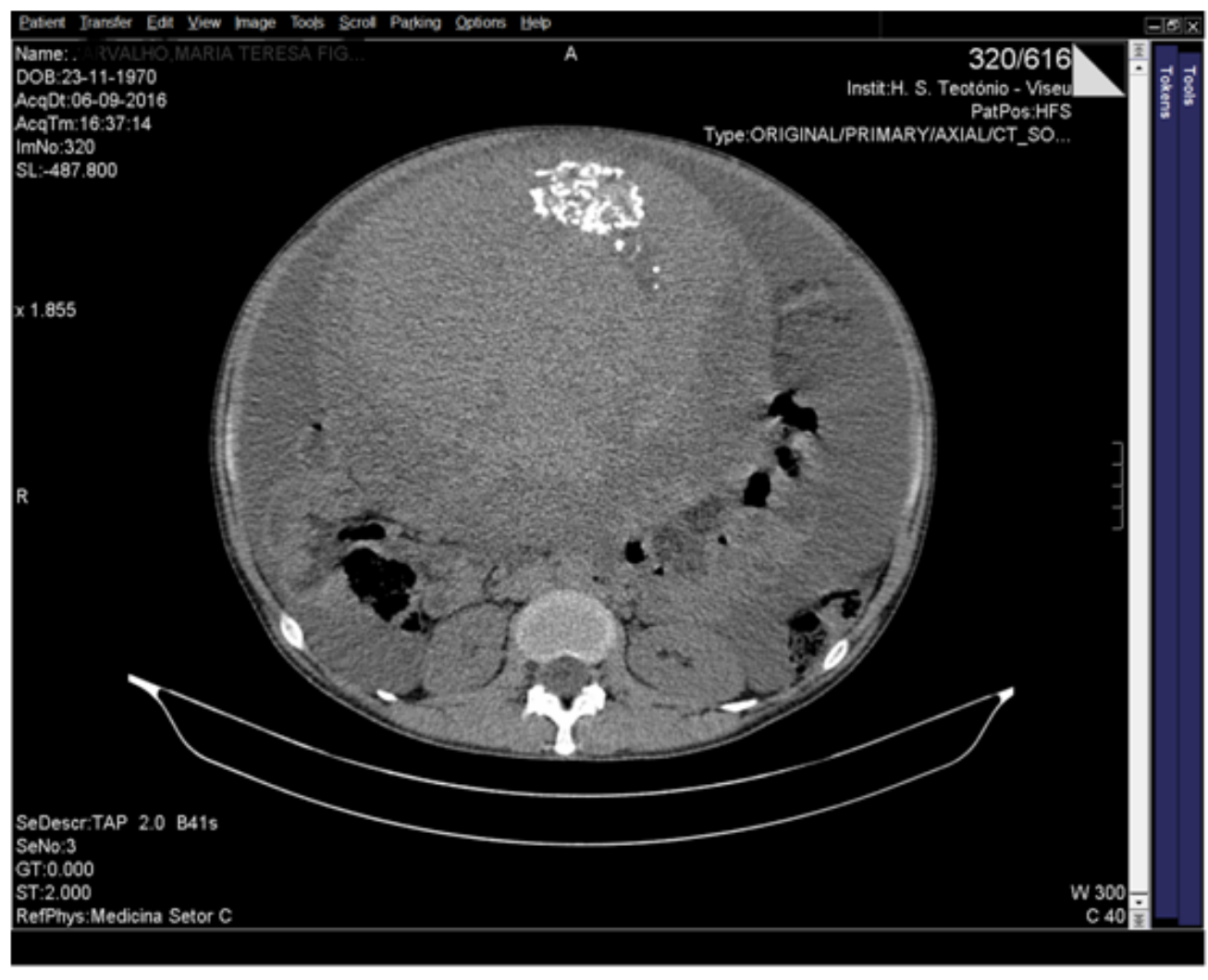

Figure 2: CT scan (transverse view) 


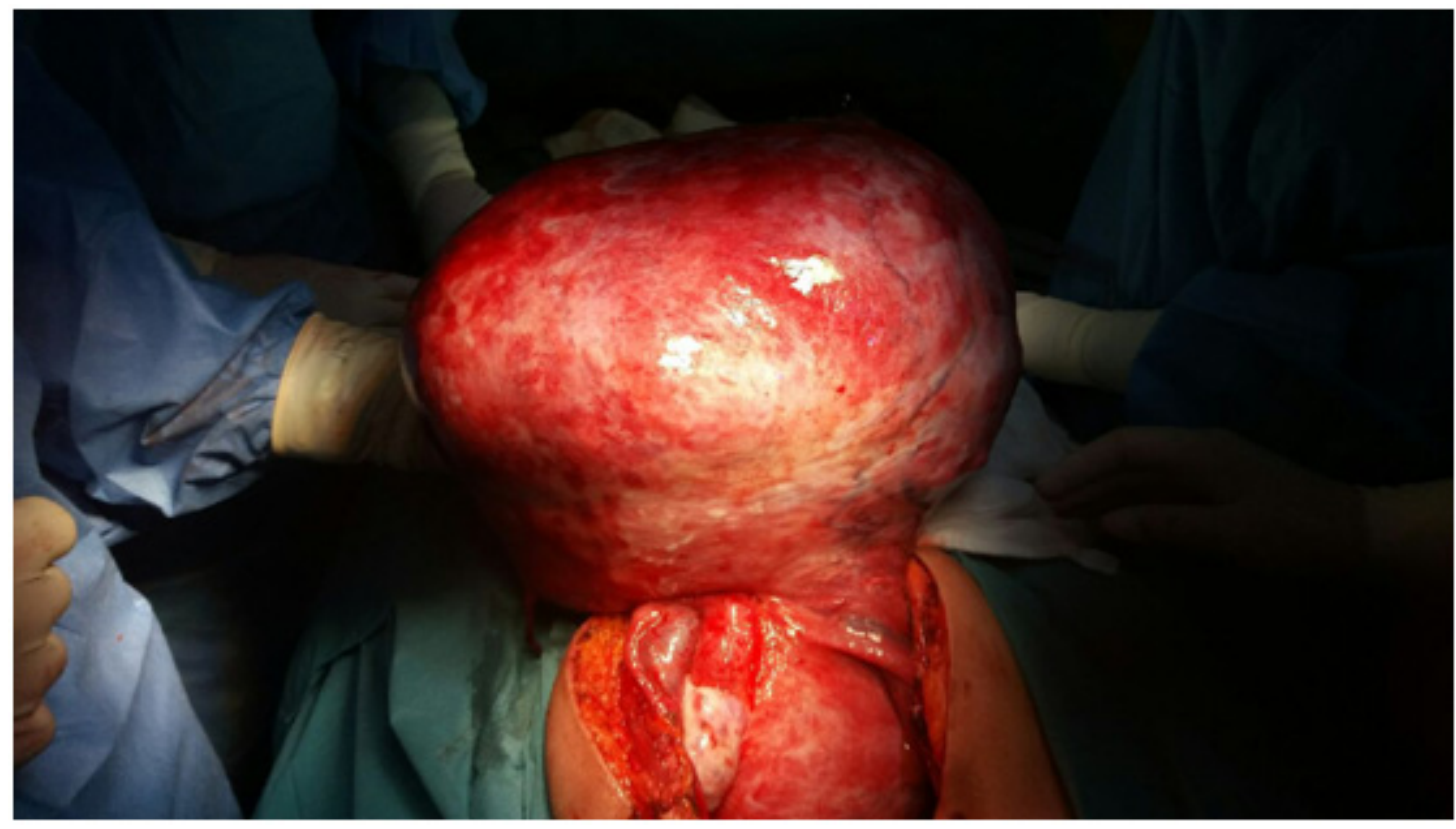

Figure 3: Uterus at laparotomy.

calcifications; right adnexa presented a uniloculated cyst measuring $6 \mathrm{~cm}$ and smooth surface; and a cervical polyp with $1.2 \mathrm{~cm}$ (figure 4 and 5 ).

The microscopy showed leiomyoma in every nodule of the body of the uterus, the bigger with calcifications and extended areas hyalinised and swollen; no necrosis or mitosis were described. Right adnexa showed a simple serous cyst.

The patient was discharged at the third day post-operatively and is currently asymptomatic, having recovered her normal weight at the follow up appointments.

\section{Discussion and Conclusions}

The differential diagnosis of an abdominalpelvic mass can be very challenging at first. Clinical evaluation, even when combined with adequate imaging and other associated complementary techniques, can be inconclusive, since benign and malignant pathologies arising from different organs as a pelvic mass may present themselves with very similar or even undistinguishable pre-operative features.

A gynecological origin may be suspected in the absence of gastrointestinal complaints, such as in the case described.

The hypothesis of ovarian cancer was included due to the association of a large pelvic mass, ascites, suspected liver metastasis and high risk ROMA. On the other hand, the abdominal CT suggested the presence of a uterine sarcoma, but the guidedbiopsy of the mass suggested the presence of leiomyoma.

The differential diagnosis of an enlarged uterus includes non-neoplastic conditions, as well as benign and malignant neoplasms such as: benign leiomyoma, leiomyoma variant, adenomyosis, uterine sarcoma, endometrial carcinoma, metastatic neoplasm (typically from another reproductive tract primary), and endometrial changes that may indirectly lead to an enlarged uterus (such as endometrial polyps, endometrial hyperplasia, hematometra and pregnancy). ${ }^{3}$

Having the ability to distinguish a leiomyoma from an uterine sarcoma pre-operatively still remains as one of the biggest challenges in women presenting an uterine mass. Taking into account the seemingly rapid growth of the tumour in this case, there was both considerable concern and belief that this was the case of malignancy. Such growth rate of uterine leiomyomas is not rare, but this quick enlargement alters the appearance of the tumor and certainly poses a diagnostic challenge, particularly in younger patients.

When dealing with a female patient with an abdominal-pelvic mass, the first step is, of course, 


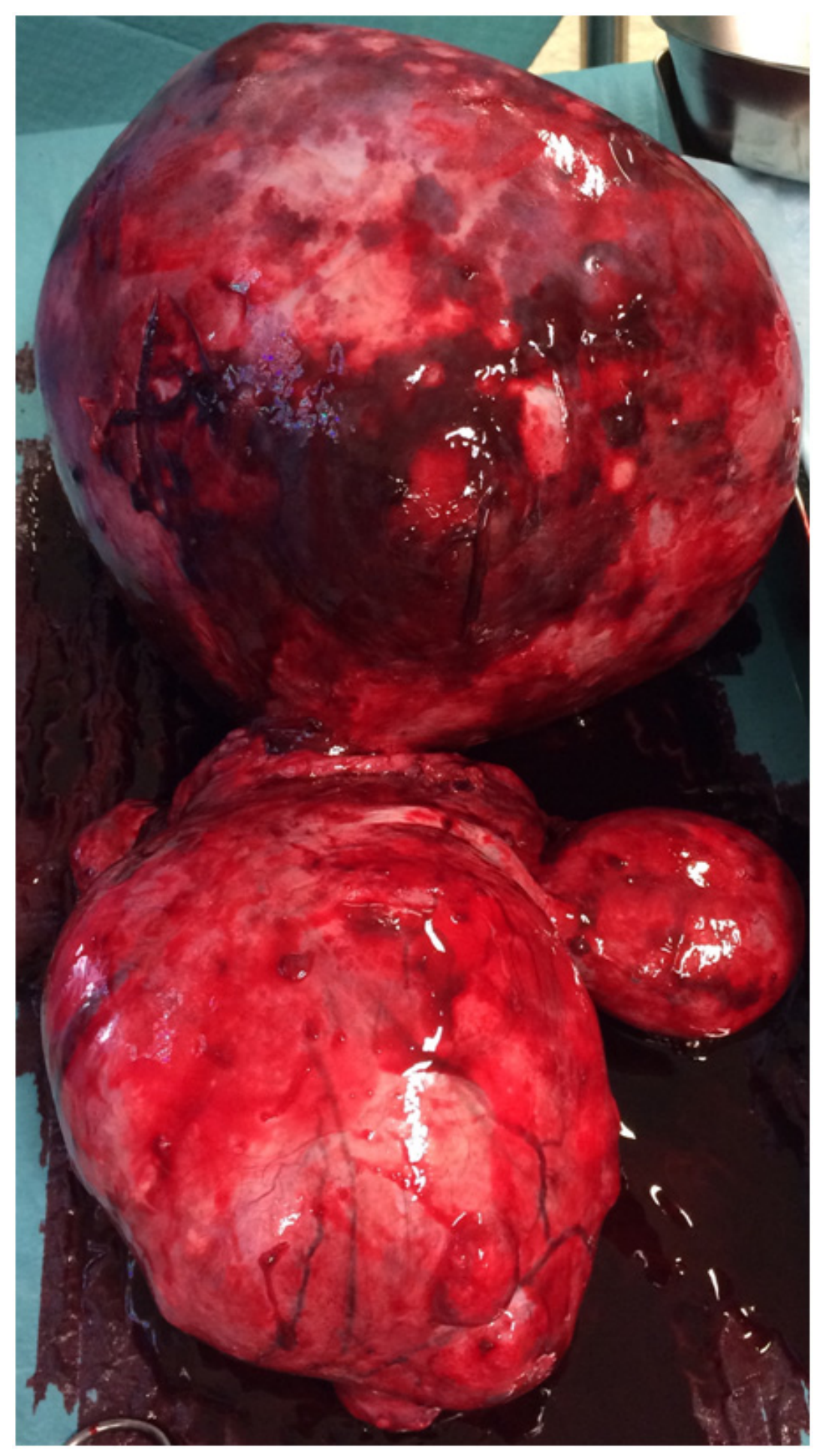

Figure 4: Picture of specimen after surgery (anterior view). 


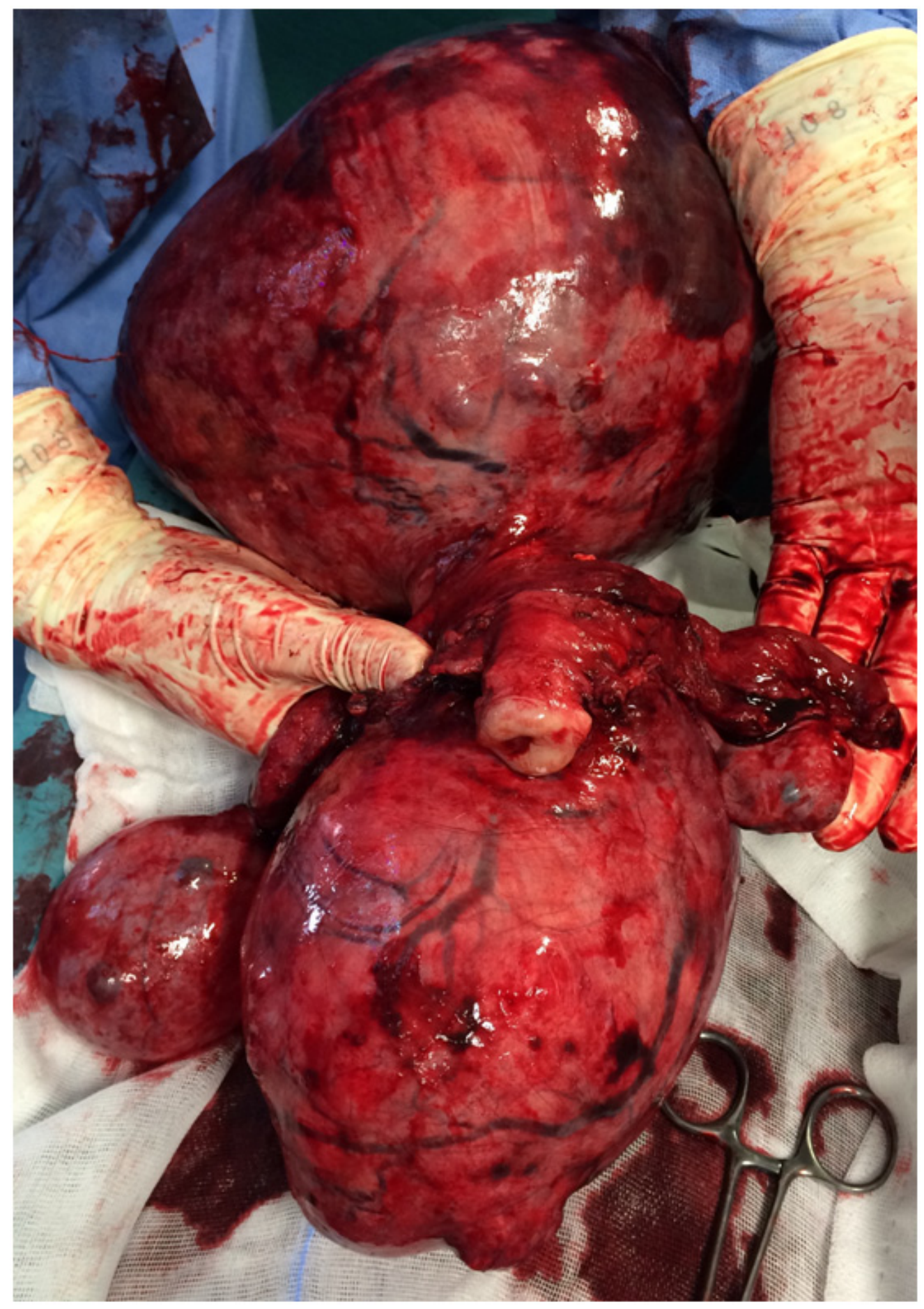

Figure 5: picture of specimen after surgery (posterior view). 
to take a good clinical history and perform a thorough physical and pelvic examination. The typical symptoms may be similar for both leiomyoma and sarcoma, and they can be unspecific, such as with abnormal uterine bleeding and/or abdominal distension. In this case, the patient presented with abdominal distension and weight loss, but no abnormal bleeding was reported.

A fixed mass is certainly more suggestive of a malignant neoplasm than a mobile mass. However, this is not pathognomonic, since on the one hand, a malignant neoplasm that has not invaded the uterine serosa may be mobile; and, on the other hand, a mass associated with endometriosis or pelvic infection may be fixed. ${ }^{3}$

Concerning diagnostic exams, there isn't yet a fully reliable pelvic imaging modality which would be able to distinguish benign leiomyomas from uterine sarcomas. ${ }^{3}$ Magnetic resonance imaging may be helpful in women in whom there is a suspicion of sarcoma; however, it does not provide a definitive diagnosis. Some data suggests that ill-defined margins are consistent with a sarcoma. ${ }^{4}$

The definitive diagnosis is only possible after obtaining an histological result and, in this case, the surgical specimen indeed confirmed the diagnosis of leiomyoma. It proved, yet again, that not everything that appears to be the case at our first evaluation is, in fact, the real diagnosis.
Some conditions are very similar, representing a true diagnostic challenge for the team. Preoperative workup provides proper guidance towards a correct diagnosis. However, all diagnostic efforts are sometimes not enough and surgery is necessary to definitively distinguish conditions that mimic others.

Special attention must be paid in order to avoid jeopardizing the patient's options whenever possible, as well as excessive time intervals between the diagnostic approach and the surgical treatment, which should optimally take place before progression of disease occurs.

\section{References}

1. Jain A, Maheshwari V, Narula V, Khan R, Sharma R. Rapidly growing giant adnexal mass in an adolescent female - a diagnostic dilemma. Acta Obstet Ginecol Port. 2013; 7: 73-6.

2. Van den Bosch T, Coosemans A, Morina M, Timmerman D, Amant F. Screening for uterine tumours. Best Pract Res, Clin Obstet Gynaecol. 2012; 26: 257-66.

3. Stewart, E. Differentiating uterine leiomyomas (fibroids) from uterine sarcomas.Uptodate.2017.Available:https:// www.uptodate.com/contents/differentiating-uterine-leiomyomas-fibroids-from-uterine-sarcomas [Acessed in 20 March 2016].

4 Schwartz LB 1 , Zawin H , Carcangiu ML , Lange R , McCarthy $\mathrm{S}$.Does pelvic magnetic resonance imaging differentiate among the histologic subtypes of uterine leiomyomata? Fertil Steril. 1998; 70: 580-7. 